\title{
A HYPERBOLIC CONSERVATION LAW AND PARTICLE SYSTEMS
}

\author{
PATRÍCIA GONÇALVES
}

\begin{abstract}
In these notes we consider two particle systems: the totally asymmetric simple exclusion process and the totally asymmetric zero-range process. We introduce the notion of hydrodynamic limit and describe the partial differential equation that governs the evolution of the conserverd quantity - the density of particles $\rho(t, \cdot)$. This equation is a hyperbolic conservation law of type $\partial_{t} \rho(t, u)+\nabla F(\rho(t, u))=0$, where the flux $F$ is a concave function. Taking these systems evolving on the Euler time scale $t N$, a Central Limit Theorem for the empirical measure holds and the temporal evolution of the limit density field is deterministic. By taking the system on a reference frame the limit density field does not evolve in time. In order to have a non-trivial limit, time needs to be speeded up and for time scales smaller than $t N^{4 / 3}$ there is still no temporal evolution. As a consequence the current across a characteristic vanishes up to this longer time scale.
\end{abstract}

\section{INTRODUCTION}

Since the early seventies Spitzer [10] has introduced to the study of interacting particle systems. The physical motivation for the study of these systems is the following. On the underlying scenario one is interested in analyzing the physical evolution of a gas or a fluid evolving in a certain volume. Two scales are considered: the macroscopic one - that describes the global motion of the physical system; and a microscopic one - that describes the movement of the molecules of the system. Due to the large number of molecules it is very difficult to give a precise description of the microscopic state of the system and the goal becomes to deduce its macroscopic evolution from the microscopic dynamics between particles. So a simplification one can do, is to assume that the motion of molecules is not deterministic but stochastic, i.e. each particle performs a random walk, subject to some local restrictions. The process describing the random motion of this collection of particles is called an interacting particle system.

In this notes, we present two examples of classical particle systems: the simple exclusion and the zero-range process, which have been very well studied in the literature. Note that in the one-dimensional case there is a classical basic coupling between them, which in some particular cases one can obtain the results for one of them by using results of the other that are easier to

Date: January 9, 2011. 
show. In this paper, we have collected some well known results proved independently, since the basic coupling does not help in this setting. The results we decide to include here are two fold. The first concerns the hydrodynamic limit: which relates the microscopic dynamics of the underlying interacting particle system to the hyperbolic conservation law that describes the macroscopic evolution of the density of particles; while the second is the Central Limit Theorem (C.L.T.) for the empirical measure: which relates the microscopic dynamics to a solution of a stochastic partial differential equation.

Here follows an outline of these notes. In the second section, we start by introducing the interacting particle systems under consideration, namely the totally asymmetric simple exclusion process and the totally asymmetric zero-range process. Then, in section three, we describe the hydrodynamic equations that govern the evolution of the density of particles, which is a hyperbolic conservation law with concave flux. For this hyperbolic law we describe its characteristics in section four. In the fifth section we state the hydrodynamic limit for these processes. Then we go further and taking the hydrodynamic time scale we state the C.L.T. for the empirical measure. The limiting process turns out to have a deterministic temporal evolution, in the sense that the limit field at time $t$ is a translation of the initial one. By removing to the system the characteristic speed the limit density field does not evolve in time and even taking a longer time scale $t N^{1+\gamma}$ with $\gamma<1 / 3$, the same behavior occurs. This is the content of the sixth section. Finally in section seven, we define the current through a fixed bond and state its convergence to the Brownian motion. We also define the current across a characteristic which vanishes for the long time scale $t N^{1+\gamma}$ with $\gamma<1 / 3$. In section eight we exhibit the dependence of the current on the initial conditions of the system. We finish the paper by presenting in section nine some concluding remarks and discussions for a future work.

\section{The Particle Systems}

In these notes we consider two different kinds of interacting particle systems, namely an exclusion type process and a zero-range process, whose dynamics can be described as follows. We start by the former. At first one fixes a probability transition rate $p(\cdot)$ on $\mathbb{Z}$ and each particle, independently from the others, waits a mean one exponential time, at the end of which being at the site $x$ jumps to $x+y$ at rate $p(y)$. In order to respect the exclusion rule, the jump succeeds as long as the destination site is empty, otherwise time restarts. This describes the general exclusion type dynamics. Now we define some restrictions on the transition rate. If $p(\cdot)$ is such that for $x, y \in \mathbb{Z}$ with $|x-y|>1$ then $p(y-x)=0$, i.e. jumps are allowed to nearest-neighboring sites, the process is called simple exclusion process. In this notes we consider $p(1)=1$ and for $n \neq 1, p(n)=0$; so that particles can only move to the right neighboring site. This process is known as the 
totally asymmetric simple exclusion process and to keep notation simple throughout the article we use the notation TASEP for it. Now we define the dynamics of the zero-range process. In this case, the main difference between last dynamics is that a particle can jump independently of the number of particles at the destination site. So, if a clock rings the particle leaves from the site $x$ to $x+y$ with rate $g(\eta(x)) p(y)$ with $g$ nondecreasing and satisfying conditions of definition 3.1 of Chap.2 of [7]. If one takes $g(n)=1_{\{n \geq 1\}}$ and $p(\cdot)$ as defined for the TASEP, then a particle at site $x$ can jump to $x+1$ at rate 1 . This process is known as the totally asymmetric zero-range process and from now on we use the notation TAZRP for it. The results we are going to present here hold for more general jump rates but in order to keep the presentation simple and clear, we opt to describe the results for this specific processes. For details on more general jump rates we refer the reader to [5] and $[6]$.

Now, we define precisely these processes by means of their generators. We define the TASEP as the Markov process $\eta_{t} \in\{0,1\}^{\mathbb{Z}}$ with generator given on local functions $f:\{0,1\}^{\mathbb{Z}} \rightarrow \mathbb{R}$ by

$$
\mathcal{L}_{T A S E P} f(\eta)=\sum_{x \in \mathbb{Z}} \eta(x)(1-\eta(x+1))\left[f\left(\eta^{x, x+1}\right)-f(\eta)\right],
$$

where

$$
\eta^{x, x+1}(z)=\left\{\begin{aligned}
\eta(z), & \text { if } z \neq x, x+1 \\
\eta(x+1), & \text { if } z=x \\
\eta(x), & \text { if } z=x+1
\end{aligned}\right.
$$

while we define the TAZRP as the Markov process $\xi_{t} \in \mathbb{N}^{\mathbb{Z}}$ with generator given on local functions $f: \mathbb{N}^{\mathbb{Z}} \rightarrow \mathbb{R}$ by

$$
\mathcal{L}_{T A Z R P} f(\xi)=\sum_{x \in \mathbb{Z}} 1_{\{\xi(x) \geq 1\}}\left[f\left(\xi^{x, x+1}\right)-f(\xi)\right],
$$

where

$$
\xi^{x, x+1}(z)=\left\{\begin{array}{ll}
\xi(z), & \text { if } z \neq x, x+1 \\
\xi(x)-1, & \text { if } z=x \\
\xi(x+1)+1, & \text { if } z=x+1
\end{array} .\right.
$$

We recall that local functions are defined on the state space of the process but depend on a configuration of the system only through a finite number of coordinates.

For the TASEP the space state is $\{0,1\}^{\mathbb{Z}}$ and configurations are denoted by $\eta$, so that $\eta(x)=0$ if the site $x$ is vacant and $\eta(x)=1$ otherwise, while for the TAZRP the space state is $\mathbb{N}^{\mathbb{Z}}$ and configurations are denoted by $\xi$, so that $\xi(x)$ denotes the number of particles at site $x$.

Now we describe a set of invariant measures for each one of the processes considered above. Fix $0 \leq \alpha \leq 1$ and denote by $\nu_{\alpha}$ the Bernoulli 
product measure on $\{0,1\}^{\mathbb{Z}}$ with density $\alpha$, i.e. its marginal at site $x$ is given by

$$
\nu_{\alpha}(\eta: \eta(x)=1)=\alpha,
$$

and for $\rho \in[0, \infty)$ denote by $\mu_{\rho}$ the Geometric product measure on $\mathbb{N} \mathbb{Z}$ with parameter $\frac{1}{1+\rho}$, i.e. for an integer $k$ the marginal at site $x$ is given by:

$$
\mu_{\rho}(\xi: \xi(x)=k)=\left(\frac{\rho}{1+\rho}\right)^{k} \frac{1}{1+\rho} .
$$

It is known that $\left(\nu_{\alpha}\right)_{\alpha}$ with $\alpha \in[0,1]$ is a family of invariant measures for the TASEP and $\left(\mu_{\rho}\right)_{\rho \geq 0}$ is a family of invariant measures for the TAZRP. We note here that these measures are homogeneous and translation invariant.

\section{Hydrodynamic EQUATIONS}

In order to keep the results presented in a condensed way, throughout the article we refer generally to a particle system $\zeta_{t}$ whose generator is denoted by $\mathcal{L}$ and with a family of invariant measures $\left(\pi_{\sigma}\right)_{\sigma}$, such that $\pi_{\sigma}$ is homogeneous, translation invariant and parameterized by the conserved quantity $\sigma$, that represents the density of particles. Whenever we want to refer to TASEP or TAZRP, $\zeta_{t}$ has to be interpreted as $\eta_{t}$ or $\xi_{t}$, the generator $\mathcal{L}$ as $\mathcal{L}_{T A S E P}$ or $\mathcal{L}_{T A Z R P}$ and the invariant measures $\left(\pi_{\sigma}\right)_{\sigma}$ as $\left(\nu_{\alpha}\right)_{\alpha}$ or $\left(\mu_{\rho}\right)_{\rho}$, as described above. We want this to be clear from now on, since the results will always be stated in terms of $\zeta_{t}$ since both the processes share mostly the same properties.

Now, we introduce the empirical measure associated to a particle system $\zeta_{t}$. For each configuration $\zeta$, denote by $\pi^{N}(\zeta, d u)$ the empirical measure given by

$$
\pi^{N}(\zeta, d u)=\frac{1}{N} \sum_{x \in \mathbb{Z}} \zeta(x) \delta_{\frac{x}{N}}(d u)
$$

and define the process of empirical measures by $\pi_{t}^{N}(\zeta, d u)=\pi^{N}\left(\zeta_{t}, d u\right)$. Here $\delta_{u}$ is the Dirac measure at $u$.

Now we give an heuristic argument to derive the conservation law that describes the evolution of the density of particles from the underlying microscopic dynamics. The processes considered here are particle-conservative in the sense that, the dynamics does not create or destroy particles, it simply moves particles according to some pre-determined rule and the number of particles is a conserved quantity.

From the classical theory of Markov processes, for a smooth test function $H$

$$
M_{t}^{N, H}=<\pi_{t}^{N}, H>-<\pi_{0}^{N}, H>-\int_{0}^{t} \mathcal{L}<\pi_{s}^{N}, H>d s
$$

is a martingale with respect to the natural filtration, where $\left\langle\pi_{t}^{N}, H\right\rangle$ denotes the integral of $H$ with respect to the measure $\pi_{t}^{N}$. For a particle system whose dynamics conserves the number of particles $\mathcal{L} \eta(x)=$ $W_{x-1, x}(\eta)-W_{x, x+1}(\eta)$, where for a site $x$ and a configuration $\eta, W_{x, x+1}(\eta)$ 
denotes the instantaneous current between $x$ and $x+1$. By a summation by parts, one can write down the martingale as

$$
M_{t}^{N, H}=<\pi_{t}^{N}, H>-<\pi_{0}^{N}, H>-\int_{0}^{t} \frac{1}{N^{2}} \sum_{x \in \mathbb{Z}} \nabla^{N} H\left(\frac{x}{N}\right) W_{x, x+1}\left(\eta_{s}\right) d s,
$$

where $\nabla^{N} H$ denotes the discrete derivative of $H$. Now we need to close the integral part of the martingale as a function of the empirical measure. Since for the models considered here the expectation of the current does not vanish, by re-scaling time by $t N$ and performing a change of variables, one gets to

$M_{t N}^{N, H}=\frac{1}{N} \sum_{x \in \mathbb{Z}} H\left(\frac{x}{N}\right)\left(\eta_{t N}(x)-\eta_{0}(x)\right)-\int_{0}^{t} \frac{1}{N} \sum_{x \in \mathbb{Z}} \nabla^{N} H\left(\frac{x}{N}\right) W_{x, x+1}\left(\eta_{s N}\right) d s$.

Now we state the notion of conservation of local equilibrium. Due to the huge number of molecules, physical systems may not be globally in equilibrium. Nevertheless fixing a small macroscopic set but huge from the microscopic point of view, by the interaction among particles, the system gets instantaneously to an equilibrium state - a local equilibrium. This means, loosely speaking, that for a site $x$ the expectation of $\eta_{t N}(x)$, is close to the expectation of $\eta(0)$ with respect to the equilibrium measure of the system, but with parameter predicted the hydrodynamic equation:

$$
E\left(\eta_{t N}(x)\right) \sim E_{\pi_{\sigma(t, x / N)}}(\eta(0)) .
$$

Taking expectation with respect to the distribution of the system at the microscopic time $t N$, to the martingale above, and since it vanishes at time 0 , it holds that

$\frac{1}{N} \sum_{x \in \mathbb{Z}} H\left(\frac{x}{N}\right)(\rho(t, x / N)-\rho(0, x / N))=\int_{0}^{t} \frac{1}{N} \sum_{x \in \mathbb{Z}} \nabla^{N} H\left(\frac{x}{N}\right) \tilde{W}(\rho(s, x / N)) d s$,

where $\tilde{W}(\sigma)=E_{\pi_{\sigma}}\left(W_{0,1}\right)$. Letting $N \rightarrow+\infty$, we identify $\rho(t, u)$ as a weak solution of the hyperbolic conservation law:

$$
\left\{\begin{array}{l}
\partial_{t} \rho(t, u)+\nabla \tilde{W}(\rho(t, u))=0 \\
\rho(0, \cdot)=\rho_{0}(\cdot)
\end{array} .\right.
$$

This partial differential equation is the hydrodynamic equation of the underlying particle system. For the TASEP, since the instantaneous current between $x$ and $x+1$ is given by $W_{x, x+1}^{T A S E P}(\eta)=\eta(x)(1-\eta(x+1))$ then $\tilde{W}^{T A S E P}(\alpha)=\alpha(1-\alpha)$ and the hydrodynamic equation becomes the inviscid Burgers equation.

$$
\partial_{t} \rho(t, u)+\nabla \rho(t, u)(1-\rho(t, u))=0 .
$$

For the TAZRP, since the instantaneous current between $x$ and $x+1$ is given by $W_{x, x+1}^{T A Z R P}(\xi)=1_{\{\xi(x) \geq 1\}}$, then $\tilde{W}^{T A Z R P}(\rho)=\frac{\rho}{1+\rho}$ and the hydrodynamic 
equation is given by:

$$
\partial_{t} \rho(t, u)+\nabla\left(\frac{\rho(t, u)}{1+\rho(t, u)}\right)=0 .
$$

In both the cases above, the mean flux of particles $\tilde{W}(\cdot)$ is a concave function.

\section{Characteristics}

In this section we describe briefly the characteristics for hyperbolic conservation laws as above. For more details we refer the reader to [8].

Consider an hyperbolic conservation law of type $\partial_{t} \rho(t, u)+\nabla \tilde{W}(\rho(t, u))=$ 0 . If $\tilde{W}(\cdot)$ is differentiable this equation can also be written as $\partial_{t} \rho(t, u)+$ $\tilde{W}^{\prime}(\rho(t, u)) \nabla \rho(t, u)=0$. A characteristic is the trajectory of a point with constant density and if one denotes by $v_{\rho_{0}}(t, u)$ the position of a point with density $\rho_{0}=\rho(0, u)$ at time $t$, then $\rho\left(v_{\rho_{0}}(t, u), t\right)=\rho_{0}=\rho(0, u)$. Taking the time derivative of last equality one gets to $\partial_{s} v_{\rho_{0}}(s, u)=\tilde{W}^{\prime}\left(\rho_{0}\right)$. Integrating from time 0 to time $t$ and noticing that $v_{\rho_{0}}(0, u)=u$, it follows that $v_{\rho_{0}}(t, u)=u+\tilde{W}^{\prime}\left(\rho_{0}\right) t$, so the characteristics of this equation are straight lines with slope $\tilde{W}^{\prime}\left(\rho_{0}\right)$.

If the initial profile is a constant function e.g. $\rho_{0}(u)=a$ for all $u \in \mathbb{R}$ and $a$ a constant, the characteristic at a point $u$ is a line with slope $\tilde{W}^{\prime}(a)$ and the characteristic speed is precisely $\tilde{W}^{\prime}(a)$. For the TASEP, starting from the equilibrium measure $\nu_{\alpha}$, which corresponds to taking the initial profile $\rho_{0}(u)=\alpha \forall u \in \mathbb{R}$, the characteristic speed is $\left(\tilde{W}^{T A S E P}\right)^{\prime}(\alpha)=(1-2 \alpha)$ while for the TAZRP starting from the equilibrium measure $\mu_{\rho}$ is $\left(\tilde{W}^{T A Z R P}\right)^{\prime}(\rho)=$ $\frac{1}{(1+\rho)^{2}}$.

\section{LAW OF LARGE Numbers FOR the EMPIRICAL MEASURE}

Let $\rho_{0}: \mathbb{R} \rightarrow[0,1]$ be an initial profile and denote by $\left(\mu_{N}\right)_{N}$ a sequence of probability measures defined on the space state of the particle system $\zeta_{t}$. Assume that a time 0 , the system starts from a initial measure $\mu_{N}$ that is associated to the initial profile $\rho_{0}$, in the following sense.

Definition 1. A sequence $\left(\mu_{N}\right)_{N \geq 1}$ is associated to $\rho_{0}$, if for every continuous function of compact support $H: \mathbb{R} \rightarrow \mathbb{R}$ and for every $\delta>0$

$$
\lim _{N \rightarrow+\infty} \mu_{N}\left[\zeta:\left|\frac{1}{N} \sum_{x \in \mathbb{Z}} H\left(\frac{x}{N}\right) \zeta(x)-\int_{\mathbb{R}} H(u) \rho_{0}(u) d u\right|>\delta\right]=0 .
$$

Note that the term on the left hand side corresponds to the integral of $H$ with respect to $\pi^{N}$, thus the above definition corresponds to asking that empirical measures at time 0 satisfy a Law of Large Numbers, namely that the sequence of random measures $\pi^{N}(\zeta, d u)$ converges in $\mu_{N}$-probability to the deterministic measure $\rho_{0}(u) d u$. The goal in hydrodynamic limit consists in showing that, if at time $t=0$ the empirical measures are associated to some initial profile $\rho_{0}$, then at the macroscopic time $t$ they are associated 
to a profile $\rho_{t}$ which is the solution of the some partial differential equation. In other words the aim is to prove that the random measures $\pi_{t N}^{N}$ converge in probability to the deterministic measure $\rho(t, u) d u$, which is absolutely continuous with respect to the Lebesgue measure and whose density evolves according to the hydrodynamic equation.

Since the work of Rezakhanlou in [9], it is known that for the TASEP starting from a sequence of measures $\left(\mu_{N}\right)_{N}$ associated to a profile $\rho_{0}(\cdot)$ and some additional hypotheses (see [9] for details) under the hyperbolic time scale $t N$ it holds that

$$
\pi_{t N}^{N} \underset{N \rightarrow+\infty}{\longrightarrow} \rho(t, u) d u
$$

in $\mu^{N} S_{N}^{T A S E P}(t)$-probability, where $\rho(t, u)$ is the entropy solution of $(3.1)$ and $S_{N}^{T A S E P}(t)$ is the semigroup associated to the generator $\mathcal{L}_{T A S E P}$. In the same work it is shown that for the TAZRP the macroscopic particle density profile in the hyperbolic scaling of time, evolves according to (3.2). We refer the reader to [9] for details on the proofs of last results.

So, the hydrodynamic limit is a Law of Large Numbers for the empirical measure associated to a particle system. If one wants to go further and show a C.L.T. for the empirical measure starting from the equilibrium state, one has to consider the density fluctuation field as defined below, see (6.1). For these processes the limit density fluctuation field at time $t$ is a translation of the initial one, whose translation velocity is given by the characteristic speed. This will be developed in the next section.

\section{Central Limit Theorem for the empirical measure}

Here we state the C.L.T. for the empirical measure for the processes considered above. Since both share the same properties, we will state the result for both as mentioned in the beginning of section three.

Let $\mathcal{S}(\mathbb{R})$ denote the Schwartz space of test functions. For a particle system $\zeta_{t}$ with invariant measure $\pi_{\sigma}$, denote by $\mathcal{Y}_{t}^{N}$ the density fluctuation field, i.e. the linear functional acting on $H \in \mathcal{S}(\mathbb{R})$ as

$$
\mathcal{Y}_{t}^{N}(H)=\frac{1}{\sqrt{N}} \sum_{x \in \mathbb{Z}} H\left(\frac{x}{N}\right)\left(\zeta_{t N}(x)-\sigma\right) .
$$

For $k \in \mathbb{N}$, let $\mathcal{H}_{k}$ be the Hilbert space induced by $\mathcal{S}(\mathbb{R})$ and $\left\langle f, g>_{k}=<\right.$ $f, K_{0}^{k} g>$, where $\left\langle\cdot, \cdot>\right.$ denotes the inner product of $L^{2}(\mathbb{R}), K_{0}=x^{2}-$ $\Delta$ and let $\mathcal{H}_{-k}$ be its dual. Denote by $D\left(\mathbb{R}^{+}, \mathcal{H}_{-k}\right)\left(\operatorname{resp} . \quad C\left(\mathbb{R}^{+}, \mathcal{H}_{-k}\right)\right)$ the space of $H_{-k}$-valued functions, right continuous with left limits (resp. continuous), with the uniform weak topology; and by $Q_{N}$ the probability measure on $D\left(\mathbb{R}^{+}, \mathcal{H}_{-k}\right)$ induced by $\mathcal{Y}^{N}$ and $\pi_{\sigma}$.

Theorem 6.1. Fix an integer $k>2$. Denote by $Q$ be the probability measure on $C\left(\mathbb{R}^{+}, \mathcal{H}_{-k}\right)$ corresponding to a stationary Gaussian process with mean 
0 and covariance given by

$$
E_{Q}\left[\mathcal{Y}_{t}(H) \mathcal{Y}_{s}(G)\right]=\chi(\sigma) \int_{\mathbb{R}} H\left(u+\tilde{W}^{\prime}(\sigma)(t-s)\right) G(u) d u
$$

for every $0 \leq s \leq t$ and $H, G$ in $\mathcal{H}_{k}$. Here $\chi(\sigma)=\operatorname{Var}\left(\zeta(0), \pi_{\sigma}\right)$. Then, $\left(Q_{N}\right)_{N}$ converges weakly to $Q$.

Last theorem holds for the TASEP and for the TAZRP, with the appropriate changes. For a proof of last result for the TASEP or TAZRP we refer the interested reader to [5] or [6], respectively. In these two cases, the limit density fluctuation field at time $t$ is a translation of the initial one, since $d \mathcal{Y}_{t}=\tilde{W}^{\prime}(\sigma) \nabla \mathcal{Y}_{t} d t$ i.e. $\forall H \in \mathcal{S}(\mathbb{R}), \mathcal{Y}_{t}(H)=\mathcal{Y}_{0}\left(T_{t} H\right)$ where $T_{t} H(u)=H\left(u+t \tilde{W}^{\prime}(\sigma)\right), T_{t}$ is a translation depending on the characteristic velocity of the system. It is easy to show that $\mathcal{Y}_{0}$ is a Gaussian field with covariance given by $\left.E_{Q}\left(\mathcal{Y}_{0}(G) \mathcal{Y}_{0}(H)\right)=\chi(\sigma)<G, H\right\rangle$.

We note here, that if one takes the system moving in a reference frame with velocity given by the characteristic speed and taking the hydrodynamic time scale, the limit density field does not evolve in time. In order to see how far this trivial temporal evolution goes, one considers the same problem by redefining the density fluctuation field on $H \in \mathcal{S}(\mathbb{R})$ as

$$
\mathcal{Y}_{t}^{N, \gamma}(H)=\frac{1}{\sqrt{N}} \sum_{x \in \mathbb{Z}} H\left(\frac{x-\tilde{W}^{\prime}(\sigma) t N^{1+\gamma}}{N}\right)\left(\zeta_{t N^{1+\gamma}}(x)-\sigma\right),
$$

where $\zeta_{t}$ is a particle system in a reference frame with velocity $\tilde{W}^{\prime}(\sigma)$ and evolving in the time scale $N^{1+\gamma}$, with $\gamma>0$. As above, let $Q_{N}^{\gamma}$ be the probability measure on $D\left(\mathbb{R}^{+}, \mathcal{H}_{-k}\right)$ induced by $\mathcal{Y}^{N, \gamma}$ and $\pi_{\sigma}$. Using the martingale approach one can derive the Boltzmann-Gibbs principle by using a multi-scale argument, see [5] for the TASEP and [6] for the TASEP. As a consequence, one can close the integral part of the martingale associated to (6.2) and state that:

Theorem 6.2. Fix an integer $k>1$ and $\gamma<1 / 3$. Let $Q$ be the probability measure on $C\left(\mathbb{R}^{+}, \mathcal{H}_{-k}\right)$ corresponding to a stationary Gaussian process with mean 0 and covariance given by

$$
E_{Q}\left[\mathcal{Y}_{t}(H) \mathcal{Y}_{s}(G)\right]=\chi(\sigma) \int_{\mathbb{R}} H(u) G(u) d u
$$

for every $s, t \geq 0$. Then, the sequence $\left(Q_{N}^{\gamma}\right)_{N \geq 1}$ converges weakly to $Q$.

This result says that speeding the process up to the time scale $N^{4 / 3}$ the limit density field does not evolve in time, i.e. $\forall H \in \mathcal{S}(\mathbb{R}) \mathcal{Y}_{t}(H)=\mathcal{Y}_{0}(H)$ and $\mathcal{Y}_{0}$ is a Gaussian field with covariance given by $E_{Q}\left(\mathcal{Y}_{0}(G) \mathcal{Y}_{0}(H)\right)=$ $\chi(\sigma)\langle G, H\rangle$. So, up to the time scale $t N^{4 / 3}$ the limit density field at time $t$ does only depend on the initial conditions of the system. 


\section{Current fluctuations}

First we introduce the notion of current of particles through a fixed bond for a particle system $\zeta_{t}$ evolving on the hyperbolic time scale $t N$. For a site $x$, let $J_{x, x+1}^{N}(t N)$ be the number of jumps from $x$ to $x+1$ during the time interval $[0, t N]$. If the number of particles is finite then one can write down

$$
J_{-1,0}^{N}(t N)=\sum_{x \geq 0}\left(\zeta_{t N}(x)-\zeta_{0}(x)\right),
$$

and in fact, it can also be written in terms of the density fluctuation field (6.1) as

$$
\frac{1}{\sqrt{N}}\left\{J_{-1,0}^{N}(t N)-\mathbb{E}_{\pi_{\sigma}}\left[J_{-1,0}^{N}(t N)\right]\right\}=\mathcal{Y}_{t}^{N}\left(H_{0}\right)-\mathcal{Y}_{0}^{N}\left(H_{0}\right),
$$

where $H_{0}$ is the Heaviside function, $H_{0}(u)=1_{[0, \infty)}(u)$. In order to give sense of last expression for an arbitrary initial condition, the density fluctuation field has to be well defined and the key point is to approximate in a proper sense the Heaviside function by a sequence of functions of compact support, we leave the details to section four of [5].

Using this relation and the C.L.T. for the empirical measure (Theorem 6.1), a C.L.T. for the current over a fixed bond can be obtained. We state the result here, but for a proof for the TASEP we refer the reader to Theorem 4.2 of [5], while for the TAZRP we refer to section 3.2 of [6].

Theorem 7.1. Fix $x \in \mathbb{Z}$ and let $Z_{t}^{N}=\frac{1}{\sqrt{N}}\left\{J_{x, x+1}^{N}(t N)-\mathbb{E}_{\pi_{\sigma}}\left[J_{x, x+1}^{N}(t N)\right]\right\}$. Then, for every $k \geq 1$ and every $0 \leq t_{1}<t_{2}<. .<t_{k},\left(Z_{t_{1}}^{N}, . ., Z_{t_{k}}^{N}\right)$ converges in law to a Gaussian vector $\left(Z_{t_{1}}, . ., Z_{t_{k}}\right)$ with mean zero and covariance given by

$$
E_{Q}\left[Z_{t} Z_{s}\right]=\chi(\sigma)\left|\tilde{w}^{\prime}(\sigma)\right| s
$$

provided $s \leq t$

Now, let the process be evolving on the longer time scale $t N^{1+\gamma}$ and moving in a reference frame with velocity $\tilde{W}^{\prime}(\sigma)$. The current through the characteristic speed $\tilde{W}^{\prime}(\sigma)$ is denoted by $J_{W_{t}^{x}(\sigma)}^{N, \gamma}\left(t N^{1+\gamma}\right)$ and corresponds to the current through the moving bond $\left[W_{t}^{x}(\sigma), W_{t}^{x}(\sigma)+1\right]$ (where $W_{t}^{x}(\sigma)=$ $\left.x+\left[\tilde{W}^{\prime}(\sigma) t N^{1+\gamma}\right]\right)$ defined as the number of jumps from $W_{t}^{x}(\sigma)$ to $W_{t}^{x}(\sigma)+1$ during the time interval $\left[0, t N^{1+\gamma}\right]$ :

$$
J_{W_{t}^{x}}^{N, \gamma}\left(t N^{1+\gamma}\right)=\sum_{y \geq 1}\left(\zeta_{t}\left(y+W_{t}^{x}\right)-\zeta_{0}(y+x)\right) .
$$

Since this current can be written in terms of the density fluctuation field (6.2) as in (7.1) and this one does not evolve in time for time scales smaller than $t N^{4 / 3}$, then is is reasonable to claim that this current should vanish. This is the content of next Propositon, which is a consequence of the C.L.T. for the empirical measure (Theorem 6.2) together with an approximation 
argument. This holds for the TASEP as well as for the TAZRP, and for details we refer the reader to [5].

Proposition 7.2. Fix $t \geq 0$, a site $x \in \mathbb{Z}$ and $\gamma<1 / 3$. Then,

$$
\lim _{N \rightarrow+\infty} \mathbb{E}_{\pi_{\sigma}}\left[\frac{1}{\sqrt{N}}\left\{J_{W_{t}^{x}}^{N, \gamma}\left(t N^{1+\gamma}\right)-\mathbb{E}_{\pi_{\sigma}}\left[J_{W_{t}^{x}}^{N, \gamma}\left(t N^{1+\gamma}\right)\right]\right\}\right]^{2}=0 .
$$

\section{Dependence on the initial COnditions}

Now, we focus on the processes evolving in the hyperbolic time scale. Since the current can be written in terms of the density fluctuation field as in (7.1) and since the limit density field at time $t$ does only depend on the initial density field, it is natural that the current should also enjoy this property. As above, let $\zeta_{t}$ denote a particle system with $\pi_{\sigma}$ as invariant measure and with characteristic speed $\tilde{W}^{\prime}(\sigma)$, then

Proposition 8.1. Fix $t \geq 0$ and a site $x$. Then,

$$
\lim _{N \rightarrow+\infty} \frac{1}{N} \mathbb{E}_{\pi_{\sigma}}\left[J_{x-1, x}^{N}(t N)-\mathbb{E}_{\pi_{\sigma}}\left[J_{x-1, x}^{N}(t N)\right]-\sum_{y=x-\tilde{W}^{\prime}(\sigma) t N}^{x-1}\left(\zeta_{0}(y)-\sigma\right)\right]^{2}=0 .
$$

For the TASEP and for $\alpha=1 / 2$, the normalized current converges to 0 in the $L^{2}\left(\mathbb{P}_{\nu_{\alpha}}\right)$-norm. This result was obtained before by Ferrari and Fontes in [3]. For a proof of last result for the TASEP we refer the reader to [5] while for the proof for the TAZRP one can follow the same arguments as done for the TASEP in [5]. This is a simple computation which is left to the reader.

This last result tells us that the flux of particles through a fixed bond $[x, x+1]$ during the time interval $[0, t N]$ does only depend on the initial number of particles in the site interval $\left[x-\tilde{W}^{\prime}(\sigma) t N, x-1\right]$ which is in accordance with the macroscopic global behavior predicted by the hydrodynamic equation.

\section{Concluding Remarks}

We finish the paper with some commentaries, questions and conjectures for a future work on the subject.

- First we note that the result of Theorem 6.2 stated with $\gamma<1 / 2$ should hold for the TASEP and TAZRP, which is predicted by the hydrodynamic equation (see [11]). On the other hand it was proved in [2] and [1] for the TASEP and TAZRP, respectively, that the order of the variance of the current across a characteristic is $t^{2 / 3}$, which translates by saying that in fact our result should hold till the time scale $N^{3 / 2}$.

- On time scale $N^{3 / 2}$, that corresponds to $\gamma=1 / 2$ there is a phase transition in the sense that fluctuations will depend on the initial conditions of the system plus the randomness of the underlying microscopic dynamics. For a physical support of this conjecture 
see [11]. With the martingale characterization approach presented above, the main difficulty is the Boltzmann-Gibbs principle which was proved to hold up to the time scale $N^{4 / 3}$, but in fact it should be valid until $N^{3 / 2}$. As a consequence, for time scales $N^{\theta}$ with $\theta<3 / 2$, the limit density field should only depend on the initial conditions of the system.

- As argued above, the current across a characteristic can formally be written in terms of the density fluctuation field, see (7.1). Since for time scales $N^{\theta}$ with $\theta<3 / 2$ the limit density field should not evolve in time, then this current should vanish. In Proposition 7.2 this result was proved until the time scale $N^{4 / 3}$. Nevertheless, we note that once the Boltzmann-Gibbs principle is proved to hold until the time scale $N^{3 / 2}$ one can obtain the same result as in Proposition 7.2 until the time scale $N^{3 / 2}$ by applying the same arguments.

- On the time scale $N^{3 / 2}$ there is a phase transition. Considering the density fluctuation field as in (6.2), it has a trivial temporal evolution until the time scale $N^{4 / 3}$, but as claimed above, it should have this behavior until $N^{3 / 2}$. On this time scale, the randomness of the dynamics should take place.

- Indeed, for $N^{3 / 2}$ the limit density field (6.2) for the TASEP should satisfy:

$\mathcal{Y}_{t}(H)=\mathcal{Y}_{0}(H)-\lim _{N \rightarrow+\infty} \int_{0}^{t} \sum_{x \in \mathbb{Z}} \nabla H\left(\frac{x-(1-2 \alpha) t N^{3 / 2}}{N}\right) \bar{\eta}_{s N^{3 / 2}}(x) \bar{\eta}_{s N^{3 / 2}}(x+1) d s$,

where for a site $y \in \mathbb{Z}, \bar{\eta}(y)=\eta(y)-\alpha$. The difficulty is to identify the limit of the integral term, nevertheless it is easy to show that it is not a martingale in $t$, but other properties are still out of reach.

- For the TAZRP, on $N^{3 / 2}$ the limit of the density field (6.2) should satisfy

$$
\mathcal{Y}_{t}(H)=\mathcal{Y}_{0}(H)+\lim _{N \rightarrow \infty} \int_{0}^{t} \sum_{x \in \mathbb{Z}} \nabla H\left(\frac{x-s N^{3 / 2} /(1+\rho)^{2}}{N}\right) V\left(\xi_{s N^{3 / 2}}(x)\right) d s,
$$

where $V(\xi(x))=1_{\{\xi(x) \geq 1\}}-\frac{\rho}{1+\rho}-\frac{1}{(1+\rho)^{2}}[\xi(x)-\rho]$.

- As a consequence for $N^{3 / 2}$ and for both processes, the current through the characteristic speed should not vanish, since one should have in a certain topology that:

$$
\frac{1}{\sqrt{N}}\left\{J_{-1,0}^{N}\left(t N^{3 / 2}\right)-\mathbb{E}_{\pi_{\sigma}}\left[J_{-1,0}^{N}\left(t N^{3 / 2}\right)\right]\right\} \sim \mathcal{Y}_{t}^{N}\left(H_{0}\right)-\mathcal{Y}_{0}^{N}\left(H_{0}\right),
$$

but now the right hand side does not vanish any longer. In fact, it was shown in [4], that for the TASEP the current through the characteristic speed on this time scale has Tracy-Widom distribution. For 
the TAZRP the result is still open. The processes considered here, belong to a universal class of processes that share the same scaling exponent and distributional properties. All the results stated above should hold for this more general class of processes.

\section{ACKNOWLEDGEMENTS}

The author thanks to "Fundação para a Ciência e Tecnologia" for the grant /SFRH/BPD/39991/2007 and to "Fundação Calouste Gulbenkian" for the Prize to the research project "Hydrodynamic limit of particle systems".

\section{REFERENCES}

[1] Balázs, M.; Komjáthy, J. (2008): Order of current variance and diffusivity in the rate one totally asymmetric zero range process Journal of Statistical Physics 133 (1), 59-78.

[2] Balazs, M., Seppalainen, T. (2009): Order of current variance and diffusivity in the asymmetric simple exclusion process. To appear in Ann. Math. and available online at http://arxiv.org/abs/math.PR/0608400.

[3] Ferrari, P., Fontes, L. (1994): Current Fluctuations for the Asymmetric Simple Exclusion Process. Annals of Probability, 22, 820-832.

[4] Ferrari, P., Spohn, H. (2006): Scaling limit for the space-time covariance of the stationary totally asymmetric simple exclusion process, Communications in Mathematical Physics, 265, 1-44.

[5] Gonçalves, P. (2008): Central Limit Theorem for a Tagged Particle in Asymmetric Simple Exclusion. Stochastic Process and their Applications, 118, 474-502.

[6] Gonçalves, P. (2009): Equilibrium Fluctuations for the Totally Asymmetric ZeroRange Process, available online at http://arxiv.org/abs/0902.3974.

[7] Kipnis, C., Landim, C. (1999): Scaling Limits of Interacting Particle Systems, Springer-Verlag, New York.

[8] Kriecherbauer, T.; Krug, J. (2008): Interacting particle systems out of equilibrium, available online at http://arxiv.org/abs/0803.2796.

[9] Rezakhanlou, F. (1991): Hydrodynamic Limit for Attractive Particle Systems on $\mathbb{Z}^{d}$. Communications in Mathematical Physics, 140, 417-448.

[10] Spitzer, F. (1970): Interaction of Markov Processes. Advances in Mathematics, 5, 246-290.

[11] Spohn, H. (1991): Large scale Dynamics of Interacting Particles. Springer-Verlag.

Centro de Matemática da Universidade do Minho, 4710-057 Braga, PortuGAL

E-mail address: patg@math.uminho.pt 